of vitamin D deficiency or insufficiency. When performing the association analysis, the vitamin D deficit/insufficiency presented an OR 12.46 (95\% $\mathrm{Cl}$ : 4.07-38.08)., $\mathrm{p}=<0.0001$ ) with the degree of activity measured with BASDAI. Regarding the comparative analysis of means between vitamin D deficiency/insufficiency and BASDAl it is $+2,253(95 \% \mathrm{Cl}: 1,241$ to $3,266, p<0.0001$ ).

Conclusion: In our study, patients with spondyloarthritis who have a vitamin D deficit correlate with presenting disease activity (BASDAl> or $=4$ ). Therefore, inflammation along with increased bone turnover and low levels of vitamin $D$ could be related to the pathophysiology of osteoporosis related to spondyloarthritis, and may adversely affect the patient's functional status and quality of life.

Disclosure of Interests: None declared

DOI: 10.1136/annrheumdis-2019-eular.4951

\section{AB1252 FUNCTIONAL STATUS CHANGES ASSOCIATED WITH INFLAMMATORY ARTHRITIS IN REPUBLIC OF MOLDOVA}

Victor Cazac, Liliana Groppa, Eugen Russu, Larisa Rotaru, Lia Chislari. State University of Medicine and Pharmacy "Nicolae Testemitanu", Rheumatology and Nephrology, Chisinau, Moldova, Republic of

Background: Inflammatory joint disease is a burden to the patient and society, due to medical costs and the impact it has on the health-related quality of life. Functional status plays a significant role in quality of life impairment.

Objectives: To study and compare functional status changes between different inflammatory joint diseases and degenerative joint disease.

Methods: 1500 patients with inflammatory joint disease and 400 patients with degenerative joint disease were included in this study. In the first group, 645 (43.0\%) were diagnosed with rheumatoid arthritis, 330 (22.0\%) - psoriatic arthritis, 100 (6.7\%) - ankylosing spondylitis, 200 (13.3\%) axial spondyloarthritis, 135 (9.0\%) - gout, 20 (1.3\%) - other crystal arthritis, $45(3.0 \%)$ - early arthritis and $25(1.7 \%)$ with other arthritis. Physical exam with functional status was assessment were performed. Descriptive statistics and Mann-Whitney $U$ tests were used to compare the study groups, as well as the subgroups within the inflammatory disease group. Results: Both groups were comparable according to sex, with a predominance of female sex $\left(\chi^{2}=86.4\right.$ and 30.74 respectively, $\left.p<0.001\right)$, and no difference in sex distribution (mean ranks of $U$ test -950.98 and 967.44, $p=0.524)$. Mean age was significantly lower in the inflammatory joint disease group (44.76 vs $60.19, \mathrm{p}<0.001)$. The Mann-Whitney $U$ test showed a significantly greater mean rank in the inflammatory disease group (999.07 vs $790.66, \mathrm{p}<0.001$ ), suggesting an overall higher functional class, and thus worse functional status in the inflammatory joint disease group. Further analysis using the same method, between subgroups in the inflammatory joint disease group, showed a higher mean rank (worse functional status) in patients with rheumatoid arthritis, when compared with psoriatic arthritis (501.95 vs 460.73, $\mathrm{p}<0.05)$ and early arthritis (352.24 and 248.83, $\mathrm{p}<0.001)$. Psoriatic arthritis showed a higher mean rank, compared to axial spondyloarthritis (282.88 vs $254.97 \mathrm{p}<0.05$ ) and early arthritis (193.34 vs 148.83, $\mathrm{p}<0.01)$. Gout patients showed a higher mean rank (worse functional status), when compared to rheumatoid arthritis (453.65 vs $377.28, \mathrm{p}<0.001)$, psoriatic arthritis (281.33 vs 213.23 , $\mathrm{p}<0.001$ ), ankylosing spondylitis (127.07 vs $105.75, \mathrm{p}<0.05)$, axial spondyloarthritis (184.67 vs $156.75, \mathrm{p}<0.01)$ and early arthritis ( 101.70 vs 56.89 , $\mathrm{p}<0.001$ ), thus making it the subgroup associated with the poorest functional status. Ankylosing spondylitis showed a higher mean rank, when compared to psoriatic arthritis $(238.13$ vs $208.64, \mathrm{p}<0.05)$, axial spondyloarthritis ( 151.75 vs $129.88, \mathrm{p}<0.05)$ and early arthritis (81.63 vs 53.83 , $\mathrm{p}<0.001)$. Axial spondyloarthritis and other crystalline arthritis subgroups both showed higher mean ranks compared to early arthritis (130.0 vs 91.89 and 43.2 vs 28.56 respectively, $p<0.01$ ), making early arthritis the subgroup associated with the best functional status. No significant differences were found when comparing rheumatoid arthritis with ankylosing spondylitis, axial spondyloarthritis and other crystalline arthritis.

Conclusion: This is the first study conducted on a national scale to assess the impact of inflammatory joint disease on functional status. Inflammatory joint disease involved a younger age group, when compared to degenerative joint disease, with a higher impact on the functional status. Interestingly, gout was associated with one of the poorest functional status, suggesting the need for a more aggressive approach toward this disease. After gout, a significantly impaired functional status was associated with rheumatoid arthritis, ankylosing spondylitis and axial spondyloarthritis, whereas early arthritis was generally associated with a better functional status, when compared to other inflammatory diseases.
Disclosure of Interests: None declared

DOI: 10.1136/annrheumdis-2019-eular.2255

\begin{tabular}{|l|l}
\hline AB1253 & DETERMINATION OF RISK FACTORS OF \\
DEVELOPMENT OF HYPERURICEMIA IN \\
PROFESSIONAL ATHLETES IN SOME SPORTS
\end{tabular}

Maria Chikina ${ }^{1}$, Maxim Eliseev ${ }^{1}$, Olga Sheliabina ${ }^{1}$, Svetlana Glukhova ${ }^{1}$, Irina Kruglova ${ }^{2}$, Evgeny Nasonov ${ }^{1}$, Alexander Lila'. ${ }^{1}$ V.A. Nasonova Research Institute of Rheumatology, Moscow, Russia, Москва, Russian Federation; ${ }^{2}$ Federal scientific and clinical center for sports medicine and rehabilitation of the Federal medical and biological Agency, Москва, Russian Federation

Background: Prevalence of hyperuricemia worldwide has been steadily increasing over the past decades and becomes higher with age.

The number of studies on the prevalence of hyperuricemia and its role in development of diseases in some social groups is low, yet there is data on its high frequency in professional athletes.

Objectives: to assess impact of certain factors upon the risk of development of hyperuricemia in professional athletes in some sports.

Methods: the study was performed as retrospective. Out of 753 athletes in six sports, 267 athletes who met the study criteria were selected. The inclusion criteria: professional athletes aged 18-40, serum uric acid level $<360 \mu \mathrm{mol} / \mathrm{l}$, according to screening tests. The exclusion criterion was refusal to undergo a thorough medical examination.

Out of 753 athletes, $228(30.3 \%)$ showed high uric acid level in their screening test, $258(34.3 \%)$ athletes refused to undergo a medical examination. 267 people were included in the study, 130 men $(48.7 \%)$ and 137 women (51.3\%), with the mean age of $24.4 \pm 5.2$ years and the average observation period of $3.0 \pm 0.3$ years. Parameters under investigation: anthropometric measurements, laboratory tests (serum levels of lactate, creatinine, crude protein, triglycerides, thy roid-stimulating hormone (TSH), AST, ALT, myoglobin, testosterone, uric acid (UA)), glomerular filtration rate (GFR) (using the MDRD formula) with subsequent statistical analysis.

Hyperuricemia was defined as SUA level $>360 \mu \mathrm{mol} / \mathrm{L}$. Hypercholesterinemia - as serum cholesterol level $>5.0 \mu \mathrm{mol} / \mathrm{L}$, hypertriglyceridemia - as serum TG level $>1.7 \mathrm{mmol} / \mathrm{L}$. High serum creatinine level as $>100 \mu \mathrm{mol} /$

Statistical analysis was conducted using the applied programs package of descriptive statistics STATISTICA 12.0 (StatSoft.Inc., USA).

Results: Hyperuricemia developed in 60 athletes (22.5\%), including 51 $(39.2 \%)$ men and $9(6.6 \%)$ women.

The frequency of risk factor detection was the following: hypercholesterinemia was detected in 91 (34\%) professional athletes, hypertriglyceridemia - in $10(3.7 \%)$, reduction in GFR $<100 \mathrm{~mL} / \mathrm{min}$ - in $46(17.2 \%), \mathrm{BMI}>25$ $\mathrm{kg} / \mathrm{m}^{2}$ - in $65(24.3 \%), \mathrm{BMl}>30 \mathrm{~kg} / \mathrm{m}^{2}-$ in $3(1.1 \%)$, arterial hypertension in $0(0 \%)$ professional athletes.

The univariate analysis showed that out of all the factors under investigation, the following factors were significantly associated with the development of hyperuricemia: male sex (OR 9.181, 95\% Cl $(4.285 ; 19.674$ $\mathrm{p}<0.001)$, hypertriglyceridemia (OR $-4.857,95 \%$ Cl $\quad(1.056 ; 22.339)$ $\mathrm{p}=0.042), \quad \mathrm{BMI}>25 \mathrm{~kg} / \mathrm{m}^{2}(\mathrm{OR}-2.470,95 \% \mathrm{Cl}(1.243 ; 4.909) \mathrm{p}=0.01)$ increased level of CPK (OR $-2.247,95 \%$ Cl $(1.230 ; 4.105) \quad p=0.008)$ Age $>25$ years was associated with lower risk of development of hyperuricemia.

According to the multifactor analysis, development of hyperuricemia is associated with male sex (OR $-13.4,95 \% \mathrm{Cl}(3.6 ; 49.6) \mathrm{p}=0.00012)$ and $\mathrm{BMI}>25 \mathrm{~kg} / \mathrm{m}^{2}$ (OR $\left.-2.4,95 \% \mathrm{Cl}(1.02 ; 5.69) \mathrm{p}=0.04\right)$.

Conclusion: hyperuricemia developed in professional athletes in $22.5 \%$ cases in 3.0 years. This study confirms high risk of development of hyperuricemia in professional athletes $(22.5 \%$ cases in the 3 -year observation period) along with low frequency of many traditional risk factors (obesity, hypercholesterinemia, hypertriglyceridemia, chronic kidney disease), which can be linked to the peculiarities of diet. This fact calls for a detailed investigation of the dietary intake of athletes with the purpose of its optimization.

Disclosure of Interests: Maria Chikina: None declared, Maxim Eliseev None declared, Olga Sheliabina Speakers bureau: Berlin Chemie Menarini, Svetlana Glukhova: None declared, Irina Kruglova: None declared, Evgeny Nasonov Speakers bureau: Pfizer, Inc., MSD, Novartis, AbbVie Inc., Celgen Corporation, Biocad, Janssen, UCB, Inc., Alexander Lila Speakers bureau: Pfizer, Inc., MSD, Novartis, AbbVie Inc., Celgen Corporation, Biocad, Janssen, UCB, Inc.

DOI: 10.1136/annrheumdis-2019-eular.3062 